\title{
Étienne Guillaume, Les sauveurs de sainte Solange : les Portugais en Berry
}

Cyril Isnart

\section{Q OpenEdition \\ 12 Journals}

Édition électronique

URL : https://journals.openedition.org/remi/9148

DOI : 10.4000/remi.9148

ISSN : $1777-5418$

Éditeur

Université de Poitiers

Édition imprimée

Date de publication : 1 décembre 2017

Pagination : 206-207

ISBN : 979-10-90426-60-3

ISSN : 0765-0752

Référence électronique

Cyril Isnart, "Étienne Guillaume, Les sauveurs de sainte Solange : les Portugais en Berry », Revue européenne des migrations internationales [En ligne], vol. 33 - n4 | 2017, mis en ligne le 01 décembre 2017, consulté le 14 avril 2022. URL : http://journals.openedition.org/remi/9148 ; DOI : https://doi.org/ $10.4000 /$ remi.9148 


\section{Notes de lecture}

\author{
Castles, Stephen \\ De Haas, Hein \\ Miller, Mark J. \\ The age of migration. International \\ Population Movements in the Modern \\ World. - Basingstoke : Palgrave MacMillan, \\ 2013. $-401 \mathrm{p}$. \\ ISBN : 978-0-230-35577-4
}

Cette cinquième édition de ce grand classique, qui est actuellement le meilleur manuel disponible, renforce encore son intérêt majeur : présenter une vue aussi exhaustive que possible des migrations internationales. Par rapport aux éditions antérieures, un chapitre important est l'attention apportée aux migrants et aux minorités dans la population active; par ailleurs, tout au long des autres chapitres est évoqué l'impact de la crise économique mondiale et en quoi elle affecte les migrations internationales depuis 2008. La perspective globale est évidemment celle, en vogue aujourd'hui, de la " migration globale " plus exactement la vision globale des migrations par-delà les spécificités continentales ou thématiques. Cette tendance actuelle permet en particulier de problématiser les conséquences des migrations sur la souveraineté des états et sur leur capacité ou non à réguler les flux migratoires; elle conduit aussi à analyser en termes de transnationalisme les formes de mobilité, sans oublier ni les pays d'origine, ni ceux d'immigration.

L'ouvrage comprend trois grandes parties. La première présente les théories et I'arrière-plan historique nécessaire à la compréhension de la situation actuelle. La seconde propose une analyse des grands ensembles migratoires, successi- vement l'Europe, le continent américain, la région de I'Asie et du Pacifique, enfin I'Afrique et le Moyen-Orient. La troisième série de chapitres s'attache aux aspects politiques, économiques et sociaux des migrations : la question de la sécurité d'abord, centrée sur le terrorisme (OCDE, Afrique et Moyen-Orient), avec une brève ouverture sur les conséquences du changement climatique sur les mouvements migratoires. La globalisation aboutit aussi à repenser le marché du travail. Sur le plan social ensuite, l'analyse se porte sur les nouvelles minorités ethniques et l'alternative entre intégration et ségrégation dans la ville globale, les enjeux liés au racisme à la participation politique aux droits linguistiques étant approfondis dans l'avantdernier chapitre. L'ouvrage se conclue par les perspectives pour le XXle siècle, avec les enjeux de coopération internationale et de gouvernance autour de l'immigration clandestine.

En résumé, la dernière édition de ce livre confirme qu'il s'agit d'un ouvrage essentiel aussi bien pour les étudiants que pour les chercheurs non spécialistes, étant entendu que les spécialistes mêmes apprécieront la hauteur de vue et la qualité des analyses. On trouvera à la fin de chaque chapitre une liste de lectures recommandées, approfondies ainsi qu'une riche bibliographie en fin d'ouvrage et un index très détaillé.

Yves Charbit Démographe, professeur émérite CEPED/Université Paris Descartes 
Étienne, Guillaume

Les sauveurs de sainte Solange : les

Portugais en Berry. - Tours : Presses universitaires François-Rabelais, 2015. - 220 p. ISBN : 978-2-86906-400-3

Comment des Portugais sont-ils devenus les principaux acteurs du renouveau d'un ancien pèlerinage au centre de la France ? Afin de comprendre cette ultime et étonnante étape de I'histoire du pèlerinage local à sainte Solange, patronne catholique du Berry, Guillaume Étienne travaille dans trois directions : une étude ethnographique et historique du pèlerinage, une approche sociologique des identités et une lecture des institutions catholiques dédiées aux migrants. Sans privilégier l'une ou l'autre de ces pistes, il conjugue au contraire les perspectives et dresse une double hypothèse convaincante : les Portugais sont devenus les " sauveurs de sainte Solange " parce que le pèlerinage leur a permis d'exprimer leur triple appartenance (portugaise, française et berrichonne) et parce que l'Église a mené une politique volontariste d'intégration des migrants dans la vie catholique.

L'ouvrage suit un plan clair. II débute par une ethnographie précise du pèlerinage tel qu'il se déroule au début des années 2010 (chapitre 1). Le jour de la fête, le lieu de culte de sainte Solange, patronne du Berry, est classiquement animé par des rituels ecclésiastiques, des actes prophylactiques et des moments non religieux. Or, pendant la procession, la messe et surtout durant l'après-midi festif, la présence de la langue, des cantiques, des costumes folkloriques et des danses portugaises permet de mesurer la place centrale des migrants portugais dans le pèlerinage. Guillaume Étienne retrace alors l'évolution de ce rituel depuis le XIXe siècle (chapitre 2). II met en évidence un effritement continu, constaté par les responsables religieux qui soulignent systématiquement - mais très classiquement - la perte de religiosité et de piété des pèlerins, au profit des amusements et des danses. Lorsqu'arrivent en force, au cours des années 1960, dans le centre de la France comme ailleurs, les ouvriers portugais et leurs familles qui fuient la dictature et la misère de leur pays, le pèlerinage semble renaître. Les migrants, facilement acceptables par les autorités religieuses et politiques en tant que bons catholiques et honnêtes travailleurs, prennent alors un rôle prépondérant qui les pare des attributs des " sauveurs de sainte Solange " (chapitre 3). Alors qu'il s'agit en premier lieu pour l'Église d'intégrer l'altérité des migrants dans la grande famille catholique, le pèlerinage constitue une occasion unique et efficace de démonstration des appartenances multiples de la population portugaise. En plus de l'affirmation des origines à travers les danses, les cantiques et les costumes exhibés pendant les rituels de la matinée comme pendant les festivités de l'après-midi, les Portugais trouvent dans le pèlerinage un espace de performances de leur appartenance à la région berrichonne et à la France, que personne ne leur renie (chapitre 4). Tout le travail de la pastorale des migrants, mise en place par I'Église catholique française, jusque dans les rituels locaux tels que celui de sainte Solange, consiste d'ailleurs à laisser les identités culturelles assez libres de s'exprimer tout en protégeant " l'idée d'Église universelle " de la corrosion de ces différences (chapitre 5).

Étrange alchimie des sens du pèlerinage - justement soulignée par l'auteur à la suite de Eade et Sallnow - qui a donc bien conduit à ce que les vertus portugaises soient consacrées, sans que le sens local ne soit effacé par les participants. La combinaison des sources et des approches qui a conduit à la compréhension du destin du pèlerinage de sainte Solange permet de rendre compte de la complexité, de l'historicité et de la dynamique des croisements entre phénomène religieux et expression des identités ethniques. Deux aspects méritent spécialement d'être soulignés afin de mesurer à la fois la singularité de l'objet de ce livre, mais également sa relative banalité dans le domaine européen. 
D’une part, la littérature anthropologique sur les usages des traditions, dans le sillon de Hobsbwam et Ranger et de Boissevain, a donné lieu à une série de monographies locales, dont chacune décrit, à l'instar du cas de sainte Solange, l'évolution contemporaine des rituels, des pèlerinages ou des traditions artisanales. Ces travaux ont très bien montré la centralité de véritables entrepreneurs des traditions qui concourent à la sauvegarde, à l'invention ou à la transformation des objets culturels, mais dont la position sociale dans le paysage local est souvent située à la marge du groupe. Érudits, universitaires, promoteurs du tourisme, hommes ou femmes politiques, néo-ruraux ou artistes, dont les liens avec les traditions peuvent se révéler assez lâches à l'analyse, ils ne se construisent pas moins comme les maillons essentiels du processus de revitalisation culturelle. On pourrait ainsi se demander dans quelle mesure les Portugais du Berry, en tant que sauveurs de sainte Solange, ne constituent pas une de ces figures d'entrepreneurs marginaux de la revitalisation des rituels. L'auteur donne plusieurs indices dans ce sens, mais le lecteur reste cependant frappé par la distance culturelle qui sépare le petit pèlerinage local des années 1960 des collages de performances folkloriques mis en place par les Portugais. Même si I'on peut classer le cas de saint Solange dans la longue série des rituels européens revitalisés, la forme hybride actuelle qui mixte le culte à un sainte locale berrichonne et le spectacle de folklore du nord du Portugal ne laisse pas d'interroger les mécanismes sociaux, apparemment peu conflictuels, de coexistence multiculturelle en Europe.

D'autre part, l'ouvrage s'intègre également dans un ensemble d'études que les spécialistes de sciences sociales dédient aux mutations du fait religieux en contexte migratoire. II en constitue sans doute un cas assez singulier : le livre de Guillaume Étienne ne traite pas des adaptations et des reformulations des représentations et des pratiques religieuses d'un groupe lors de son installation dans un autre lieu, mais de l'appropriation, par un groupe migrant, d'un rituel de la société qui le reçoit. Entre la circulation européenne des images de la vierge de Guadalupe pour les Mexicains, la performance des rituels du Saint-Esprit par les Portugais au Brésil et aux États unis ou les syncrétismes des religions afro-brésiliennes dans les diasporas des anciens esclaves noirs, le renouveau de sainte Solange illustre une forme de circulation religieuse d'un genre particulier. La mobilisation de ces études sur le fait religieux en migration aurait pu apporter une dimension plus générale et plus comparative au projet de Guillaume Étienne et montrer I'originalité de la démarche portugaise dans le Berry. Par ailleurs, une analyse de la dimension matérielle de la migration et de la religion, qui étudie par exemple les objets rituels, les costumes ou la nourriture qui sont utilisés pendant le pèlerinage aurait sans doute donné l'occasion à l'auteur de décrire comment les transformations matérielles du religieux issues de la migration, dans le cas singulier de sainte Solange, répondent à celles qui ont lieu plus classiquement.

Mais tel n'était sans doute pas le propos de l'auteur et l'on ne saurait lui en faire grief. Guillaume Étienne a su rendre compte sociologiquement et ethnographiquement d'un fait localisé, dans son inscription communautaire, régionale et nationale et dans sa triple dimension rituelle (à travers l'évolution d'un pèlerinage), morale (dans l'analyse des conditions d'accueil des migrants) et ecclésiastique (grâce à une revue des textes et des pratiques de l'Église). Le livre intéressera les sociologues, les anthropologues ou les géographes des migrations et de la religion qui pourront y trouver des éléments descriptifs et analytiques solides.

Cyril Isnart

Anthropologue, chargé de recherche IDEMEC/Aix-Marseille Université 
Demart, Sarah (dir.)

Abrassart, Gia (dir.)

Créer en post-colonie. 2010-2015. Voix et dissidences Belgo-Congolaises. - Bruxelles :

Bozar et Africalia, 2016. - $329 \mathrm{p}$.

ISBN : 978-9-074-81649-6

En 2013, Sarah Demart, alors chercheure post-doctorale au CEDEM à I'Université de Liège co-organisait un colloque intitulé Arts de diaspora congolaise (République démocratique du Congo) en Belgique : imaginaire et relations postcoloniales. Cet ouvrage qui rassemble plus de cinquante auteurs et de nombreuses photos de grande qualité est la trace de ce colloque qui avait réuni la crème des penseurs, activistes et artistes postcoloniaux congolais et belgo-congolais des différentes régions du pays.

Cet ouvrage n'est pas à proprement parler un livre académique. II est publié conjointement par Bozar, le Palais des Beaux-Arts de Bruxelles, une des institutions culturelles les plus prestigieuses du pays et par Africalia, une association active dans le domaine de la coopération culturelle avec l'Afrique et dirigé par Sarah Demart et Gia Abrassart. II rassemble une cinquantaine de contributions signées par des chercheurs, des artistes, des militants associatifs sur des sujets extrêmement divers relatant la variété de la pensée et de l'action postcoloniale en Belgique. Le lecteur ne trouvera pas dans ce livre d'avancée théorique majeure sur le postcoIonialisme. Celles et ceux qui considèrent que la pensée postcoloniale n'est qu'un " carnaval académique " porté par une élite africaine privilégiée trouveront dans l'ouvrage des arguments en faveur de leur thèse. Celles et ceux qui s'inscrivent dans ce courant de pensée et d'action postcoloniale aussi. Car ce livre les " représente ", au sens que le hip-hop donne à ce terme.

Quant aux chercheur.e.s intéressé.e.s par toutes les réalités migratoires, diasporiques et post-migratoires, elles et ils trouveront dans ce document une foule d'informations très intéressantes et pertinentes sur des questions telles que la contribution culturelle des immigrés et de leurs descendants, en général, Congolais en particulier. Ce livre donne à réfléchir sur les relations entre les pratiques artistiques, l'immigration et les diasporas. II montre comment les Arts peuvent être un vecteur de mobilisation et de résistance à l'ère de la post-vérité. Ce livre à multiples facettes est donc susceptible d'intéresser des publics multiples. II indique aussi que quelque chose a clairement commencé à changer dans le Royaume de Belgique en ce qui concerne la reconnaissance de I'héritage colonial et peut-être même l'entrée très lente dans une ère post-postcoloniale. Que des artistes issus de la diaspora africaine entrent de plus en plus fréquemment aux Bozar, comme on dit à Bruxelles, est à cet égard loin d'être anodin. Le fait que l'ouvrage ait été coordonné par une chercheure "blanche " et une journaliste " métisse " non plus.

Marco Martiniello Sociologue, directeur de recherche au FRS-FNRS

CEDEM-FASS/Université de Liège

\section{Dukic, Suzana}

L'immigration en Languedoc-Roussillon du $X I X e$ siècle à nos jours. Synthèse historique, enjeux contemporains. - Canet : Éditions Trabucaire, 2014. - $174 \mathrm{p}$. ISBN : 978-2-84974-201-3

Le présent ouvrage qui résulte d'une commande officielle offre une synthèse commode sur deux siècles d'immigration dans la région Languedoc-Roussillon. Le plan, rigoureusement chronologique, rappelle d'abord les mobilités anciennes, la présence de marchands et d'intellectuels étrangers dès le Moyen Âge, de banquiers et de négociants d'Europe du Nord, de pêcheurs catalans, de mineurs flamands et allemands à l'époque moderne, des premiers réfugiés politiques espagnols au $\mathrm{XIXe}$ siècle. 
L'étude gagne en précision pour la période postérieure à 1850 . La population étrangère devient alors plus nombreuse : elle passe de 8664 personnes en 1851 à 19677 en 1911, soit, à cette date, 3 \% de la population totale de la région. Les Espagnols tiennent de loin le premier rang, suivis par les Italiens. À partir de 1880, la concurrence sur le marché de l'emploi nourrit une fréquente xénophobie qui culmine lors du massacre des Italiens à Aigues-Mortes en 1893.

Pendant la Grande Guerre, I'appel à la main-d'œuvre étrangère s'accélère. Dans les années 1920, le déficit démographique de la région renforce le mouvement. Les immigrés constituent $8 \%$ des actifs du Languedoc-Roussillon ; les femmes représentent un effectif stable de $40 \%$ des nouveaux venus. Les Espagnols et les Italiens, toujours majoritaires, occupent, comme avant 1914, une place centrale dans l'agriculture, tandis que les mines du Gard font appel à des Polonais, des Tchèques et des coloniaux. La crise des années 1930 et le renforcement de la xénophobie - le mot " espagnols " devient une injure - entraînent une rétractation des effectifs : 159213 individus en 1931 et 129088 en 1936, cela malgré l'attitude des employeurs qui essaient de limiter les mesures de contingentement de la main$d^{\prime} œ u v r e$ étrangère.

À partir de1936 la situation se dramatise : arrivée de groupes de réfugiés espagnols dès le début de la guerre civile, avant la grande Retirada au début de 1939, jetant les réfugiés par centaines de mille sur les toutes de l'exil ; création de nombreux camps de concentration improvisés où les internés vaincus sont soumis à un régime très dur ; mise au travail obligatoire des réfugiés dès avril 1939, formule reprise et amplifiée par le régime de Vichy ; déportation des juifs étrangers : neuf convois quittent Rivesaltes pour Auschwitz ; internement des travailleurs indochinois à Agde. Dans le même temps, la résistance étrangère $s^{\prime}$ organise.
Les combattants espagnols bien préparés par la guerre civile y tiennent une place éminente : réseaux de passage clandestins à travers les Pyrénées, sabotages, évasion de prisonniers, maquis allemands antinazis dans les Cévennes se multiplient. De la sorte, les étrangers jouent un rôle très actif dans la Libération.

Après la guerre, le mouvement d'immigration repart à la hausse : 114467 personnes en 1946 et 147348 en 1968, soit $8,6 \%$ de la population totale. Les Espagnols tiennent toujours la première place, tandis que les Italiens et les Polonais refluent. Les Portugais deviennent plus nombreux, de même que les Algériens. Plus de $55 \%$ des étrangers sont toujours employés dans le secteur primaire, majoritairement comme ouvriers agricoles. Mais la place de cette activité recule tandis que, avec l'arrivée des Portugais, se fortifie le BTP avec 10,4 \% des étrangers en 1954 et $32,4 \%$ en 1975 . Certains secteurs comme la viticulture et la riziculture restent très dépendants des saisonniers espagnols, souvent clandestins. Des camps de harkis sont créés, notamment à Saint-Mauricel'Ardoise.

Après 1974, la fin desTrente Glorieuses, les naturalisations et les décès entraînent un recul des effectifs : 142923 personnes en 1975 (8\%) et 131493 en 1999 (5,7\%). Les Ibériques, jusque-là majoritaires, sont progressivement remplacés par les Marocains, notamment dans I'agriculture. Le chômage frappe durement les immigrés : en 1982, le taux est de 11,2\% chez les Français et de 18,9 \% chez les étrangers. La proportion des femmes reste stable autour de $44 \%$. Dans la dernière période arrivent des résidents aisés, plutôt âgés et diplômés, venant d'Allemagne, de Belgique, de Suisse, ainsi que des étudiants et de jeunes mineurs isolés originaires du Maghreb. Les difficultés économiques entretiennent toujours la xénophobie, mais les Espagnols, désormais bien connus et enracinés, prennent figure d'exemples d'intégration. Comme dans 
d'autres régions, la gestion des lieux de culte musulman est instrumentalisée et constitue un enjeu de luttes de pouvoir. Le livre s'achève sur quelques considérations relatives à la construction de la mémoire de l'immigration et à une analyse de l'éventuelle ethnicisation des cultures.

Le livre apparaît relativement rapide sur les comportements culturels, surtout dans les groupes étrangers, les processus d'intégration, les aspects identitaires. C'est sans doute en raison du nombre réduit de pages qui lui étaient imparties que Suzana Dukic a voulu produire une courte synthèse mettant l'accent sur la dimension démographique et économique. Cette mise au point toujours claire et enrichie d'une importante bibliographie rendra de grands services. Elle offre l'intéressant exemple d'une originale immigration agricole qui resta majoritaire dans le long terme.

Ralph Schor Historien, professeur émérite Université de Nice-Sophia-Antipolis 